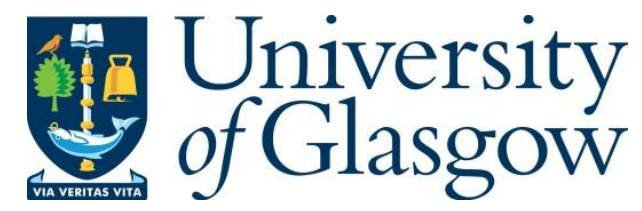

Romero, F. D. et al. (2017) Redox-controlled potassium intercalation into two polyaromatic hydrocarbon solids. Nature Chemistry, 9(7), pp. 644-652. (doi:10.1038/nchem.2765)

This is the author's final accepted version.

There may be differences between this version and the published version. You are advised to consult the publisher's version if you wish to cite from it.

http://eprints.gla.ac.uk/140279/

Deposited on: 26 April 2017

Enlighten - Research publications by members of the University of Glasgow http://eprints.gla.ac.uk 


\title{
Redox-controlled potassium intercalation into two polyaromatic hydrocarbon solids
}

\author{
F. Denis Romero1, M. J. Pitcher'1, C. I. Hiley¹, G. F. S. Whitehead'1, S. Kar'1, A. Y. Ganin'1 ${ }^{1}$, D. \\ Antypov$^{1}$, C. Collins ${ }^{1}$, M. S. Dyer ${ }^{1}$, G. Klupp², R. H. Colman'2, K. Prassides ${ }^{3,4 *}$ and M. J. \\ Rosseinsky $^{1^{*}}$
}

${ }^{1}$ Department of Chemistry, University of Liverpool, L69 7ZD, UK

2Department of Chemistry, Durham University, Lower Mountjoy, South Road, Durham DH1 3LE, UK

${ }^{3}$ WPI-AIMR, Tohoku University, 2-2-1 Katahira, Aoba-ku, Sendai 980-8577, Japan

4Japan Science and Technology Agency, ERATO Isobe Degenerate $\pi$-Integration Project, Tohoku University, Sendai 980-8577, Japan

tPresent address: School of Chemistry, University of Glasgow, Joseph Black Building, University Avenue, Glasgow G12 8QQ, UK

*Authors to whom correspondence should be addressed.

Email: m.j.rosseinsky@liverpool.ac.ukand k.prassides@wpi-aimr.tohoku.ac.jp

\begin{abstract}
Alkali metal intercalation into polyaromatic hydrocarbons (PAHs) has been studied intensely following reports of superconductivity in a number of potassium- and rubidium-intercalated materials. There are however no reported crystal structures to inform understanding of the chemistry and physics because of the complex reactivity of PAHs with strong reducing agents at high temperature. Here we present the synthesis of crystalline $\mathrm{K}_{2}$ Pentacene and $\mathrm{K}_{2}$ Picene by a solid-solid insertion protocol that uses potassium hydride as a redox-controlled reducing agent to access the PAH dianions, enabling determination of their crystal structures. In both cases, the inserted cations expand the parent herringbone packings by reorienting the molecular anions to create multiple potassium sites within initially dense molecular layers, and thus interact with the PAH anion $\pi$-systems. The synthetic and crystal chemistry of alkali metal intercalation into PAHs differs from that into fullerenes and graphite, where the cation sites are pre-defined by the host structure.
\end{abstract}

Reaction of alkali and alkaline earth metals with carbon-based molecular solids has been extensively studied in the search for novel magnetic and electronic properties, with a particular focus on superconductivity. ${ }^{1-6}$ For example, alkali metal intercalation into solid $\mathrm{C}_{60}$ produces $\mathrm{A}_{3} \mathrm{C}_{60}$ superconductors, with $T_{\mathrm{c}}$ as high as $38 \mathrm{~K}$ for $\mathrm{Cs}_{3} \mathrm{C}_{60}$ at 7 kbar. ${ }^{6}$ In these materials, cations occupy the interstitial voids that already exist in the host e.g., in the fcc lattice of $\mathrm{C}_{60}$ (Figure 1). ${ }^{7}$ Reaction of potassium with picene $\left(\mathrm{C}_{22} \mathrm{H}_{14}\right)$, a phenacene composed of five fused benzene rings, has been reported to afford superconductivity at $18 \mathrm{~K} .^{8}$ Superconductivity in other alkali-metal polyaromatic hydrocarbons (PAHs) was subsequently reported in phenanthrene-, dibenzopentacene- and coronene-based materials, with the highest reported $T_{\mathrm{c}}$ of $33 \mathrm{~K}$ claimed 
in potassium-doped 1,2:8,9-dibenzopentacene. ${ }^{9-11}$ Despite the significant interest in these materials, to date no crystal structure has been determined for any of the alkali-metal PAH systems. This structural information is a prerequisite for materials design and for understanding of both physical properties and reaction chemistry.

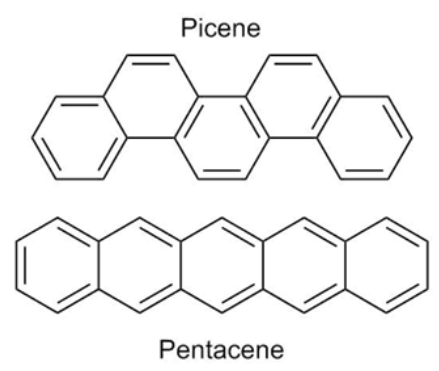

Picene, like many other PAHs (including molecules such as phenanthrene ${ }^{12}$ which is reported to afford superconductivity on cation insertion), crystallises in the herringbone structure, ${ }^{13}$ with layers consisting of two parallel one-dimensional chains of molecules with opposing inclinations defined by an intermolecular angle, $\omega$, of $57.89(7)^{\circ}$ (Supplementary Figure 1). ${ }^{14}$ The largest voids in the structure are located between the picene layers, adjacent to the saturated $\mathrm{C}-\mathrm{H}$ bonds and far from the electron density of the PAH $\pi$-systems (Figure 1a). This contrasts with $\mathrm{C}_{60}$, where the octahedral and tetrahedral voids in the fcc lattice are adjacent to the conjugated $\pi$-electron system (Figure $1 b)^{15}$.

Pentacene is the linear isomer of picene, and also adopts the herringbone structure, ${ }^{16}$ with $\omega=$ $52.9^{\circ}$ (Supplementary Figure 1). Bulk $K_{x} P$ entacene $(x=1,2$ and 3.6) has previously been reported without structural characterisation, ${ }^{17-19}$ and metallic conductivity has been reported in potassium-doped pentacene thin films. ${ }^{20-24}$ As pentacene is more readily available than picene, we chose initially to address the synthesis of potassium-intercalated pentacene samples suitable for structural analysis, before applying these protocols to the picene system.

\section{Results and Discussion}

\section{Synthesis of $K_{2}$ Pentacene}

Initial attempts to synthesise potassium-intercalated pentacene by direct reaction with potassium metal failed to produce single phase materials due to the partial or complete decomposition of the pentacene moieties: both vapour-solid and liquid-solid reactions were attempted at temperatures of $190-240{ }^{\circ} \mathrm{C}$, and showed a strong tendency to produce $\mathrm{KH}$ (a signature of PAH decomposition), in addition to crystalline products that could not be indexed to a single phase (see Supplementary Section 1 and Supplementary Figures 2 and 3). This led us to investigate $\mathrm{KH}$ as a thermodynamically milder, solid-state reducing agent and potassium source. The hydride anion is a less powerful reducing agent than potassium metal $\left(\mathrm{K}^{+} / \mathrm{K}=-2.93\right.$ $\mathrm{V}$, and $\left.\mathrm{H}_{2} / \mathrm{H}^{-}=-2.23 \mathrm{~V}\right),{ }^{25}$ but is still sufficiently reducing to access PAH anions. ${ }^{26}$ Solid-state reaction between intimately mixed powders of pentacene and $\mathrm{KH}$ under vacuum at $\mathrm{K}_{x}$ Pentacene compositions $1 \leq x \leq 4$ were performed at temperatures of $180-300{ }^{\circ} \mathrm{C}$ (see Methods). The starting composition was found to have a strong influence on the resulting phase assemblage: 
reactions produced a mixture of a new crystalline phase with either unreacted pentacene (when $x<2$ ) or KH (when $x>2$ ) for all temperatures (Supplementary Figure 4). However, the composition $x=2$ appears to be free of starting materials when reacted at $280{ }^{\circ} \mathrm{C}$ (Figure $2 \mathrm{a}$, Supplementary Figures 4 and 5). Reaction at temperatures of $280-300{ }^{\circ} \mathrm{C}$ for extended periods promoted the formation of a secondary crystalline phase, whose Bragg reflections overlap heavily with those of the majority phase (Supplementary Figure 6), therefore reaction times were kept to a minimum (see Methods). Raman and infra-red absorption (IR) spectroscopy confirm the presence of pentacene anions: in the Raman spectra, all features above the detection limit can be assigned to pentacene-derived modes (Supplementary Figure 7), consistent with the presence of only a single charge state of pentacene with no amorphous impurities. Comparison with the Raman spectrum of pentacene reveals a shift of the major features to lower energy upon reduction (Figure $2 \mathrm{~b}$ and Supplementary Figure 7), consistent with a weakening of the $\mathrm{C}-\mathrm{C}$ bonds due to population of antibonding orbitals. The IR spectrum of $\mathrm{K}_{2}$ Pentacene (Figure 2c) contains no signals corresponding to reported spectra of neutral or monoanionic pentacene, ${ }^{27}$ indicating that the pentacene charge state is greater than one.

High resolution synchrotron powder X-ray diffraction (SXRD) data from $\mathrm{K}_{2}$ Pentacene reveal that the most intense Bragg peaks have well defined shoulders (Figure 3a, b inset), which are not apparent from laboratory powder X-ray diffraction (PXRD). The positions and intensities of these shoulders correspond to a secondary crystalline $\mathrm{K}_{x}$ Pentacene phase, which becomes the majority phase when the samples are subjected to extended annealing times above $280{ }^{\circ} \mathrm{C}$ (Supplementary Figure 6). This suggests that $\mathrm{K}_{2}$ Pentacene begins a slow decomposition to produce a secondary crystalline phase of similar composition under the synthetic conditions required for phase formation, though the best samples have a sufficiently small fraction of the second phase to be undetectable by laboratory PXRD. As the main phase dominates the observed intensities in the SXRD pattern, we undertook $a b$ initio structure solution in order to determine its essential structural features (see Methods and Supplementary Section 2), producing a rigid body model which provided a good fit to the main features of the diffraction pattern (Figure 3a), comparable to that obtained from a single phase Le Bail fit (Supplementary Figure 8). The un-modelled shoulder features were then fitted as a secondary Le Bail phase, resulting in an excellent fit to the data which is dominated by the $\mathrm{K}_{2}$ Pentacene Rietveld phase (Figure 3b), and comparable to that obtained from a two-phase Le Bail fit (Supplementary Figure 8). The similarity of the unit cell parameters between the majority Rietveld phase and the minority Le Bail phase (see Supplementary Table 1) suggests their structures are closely related. The refined Rietveld model obtained from this two phase refinement (Supplementary Table 1) is similar to that obtained from a single phase Rietveld refinement (Supplementary Table 2), but has more accurate refined lattice parameters which are otherwise biased by the unmodelled secondary phase.

\section{Structure of $\mathrm{K}_{2}$ Pentacene}

Potassium insertion to afford $K_{2}$ Pentacene expands the original unit cell ${ }^{28}$ by $15 \%$ per pentacene moiety, driving a reorientation of the molecules that creates pockets within the layers to permit cation incorporation and interaction with the dianion $\pi$-systems. The C-C bond

distance of the pentacene relaxes to 1.5121(4) Å ( $v s$ a mean C-C distance of 1.41(3) $\AA$ in pristine 
pentacene ${ }^{16}$ ), which is consistent with the population of anti-bonding orbitals in the di-anion. The herringbone packing of the pentacene array is maintained, but is expanded by the pentacene reorientation, with $\omega$ increasing from $52.9^{\circ}$ in pristine pentacene ${ }^{16}$ to $90.0(4)^{\circ}$ in $\mathrm{K}_{2}$ Pentacene, as shown in Figure 3c (see Supplementary Section 3, Supplementary Table 3 and Supplementary Figure 9 for extended structure comparison and metrics). The two crystallographically distinct pockets, which have essentially the same shape but a small difference in absolute volume, are each defined by four pentacenes from the layer itself and capped at either end by a pentacene from each adjacent layer (Figure 3d). The two cations per pocket are distributed over four sites in a disordered manner that produces K...K separations of no closer than 5.31(4) $\AA$, because the closest pairs of sites have mean occupancies of no higher than $50 \%$ (Supplementary Table 1). The zig-zag width of a pentacene molecule gives the pockets an undulating cross-section (Figure 3e) whereby the only voids of sufficient size to accommodate potassium cations are adjacent to the narrowest part of the pentacene molecules.

The molecular reorientation that creates the pockets strengthens supramolecular $\mathrm{C}-\mathrm{H} \ldots \pi$ interactions, as the $\mathrm{C}-\mathrm{H}$ bonds on the long edge of the pentacene molecule become both closer to and better aligned with the electron density of the aromatic rings of neighbouring molecules: it is established for single $\mathrm{C}-\mathrm{H}$... arene interactions that perpendicular approach of the $\sigma$ bond over the ring centroid is favourable. ${ }^{29}$ In pristine pentacene, these bonds are directed towards the perimeter of neighbouring molecules (corresponding to a mean offset $\delta$ of the molecular centroids of $1.075(18) \AA$ ) at an acute angle $\omega$ (Supplementary Figure 10), whereas in $\mathrm{K}_{2}$ Pentacene, they align almost perpendicular to the aromatic rings of their neighbours and more closely to the ring centres ( $\delta$ decreases to $0.15(9) \AA)$. The mean C-H...aromatic plane distance, $d$, shortens from $2.80(5) \AA$ in pristine pentacene to 2.47(5) $\AA$ in $K_{2}$ Pentacene (Supplementary Figure 11), consistent with the increased electron density in the $\pi$-system strengthening the interaction in the salt. The steric influence of the hydrogen atoms involved in the $\mathrm{C}-\mathrm{H} . . . \pi$ interactions with adjacent molecules defining the pocket causes the inserted cations to be displaced from the centroids of the aromatic rings (Figure $3 \mathrm{c}$ and Supplementary Figure 11). Consequently, $\eta^{6}$ coordination of potassium by the aromatic rings is not possible, and instead the partially occupied cation locations correspond to the widest points of the pockets (as illustrated by Figure 3d, e), with a closest K...C contact of 2.96(7) $\AA$, interacting with the edges of the pentacene molecules. The influence of the herringbone packing on the cation locations in $\mathrm{K}_{2}$ Pentacene contrasts with the cation environments found in potassium acene salts such as $\mathrm{K}_{2}\left(\mathrm{C}_{10} \mathrm{H}_{8}\right)_{2}(\mathrm{THF})^{30}$ and $\mathrm{K}_{2}\left(\mathrm{C}_{14} \mathrm{H}_{10}\right)(\mathrm{THF})_{3},{ }^{31}$ which are synthesised in solution. In these compounds, the mode of $\mathrm{K}^{+}$coordination is not restricted by the need to retain the $\mathrm{C}-\mathrm{H}$...arene interactions of a pre-existing host network and is given extra flexibility by the incorporation of solvent moieties. This results in $\eta^{6}$ coordination of $\mathrm{K}^{+}$by the acene anions in a relatively low density (non-herringbone) array. The solution-synthesised caesium phenanthrene salts $\mathrm{Cs}\left(\mathrm{C}_{14} \mathrm{H}_{10}\right)$ and $\mathrm{Cs}_{2}\left(\mathrm{C}_{14} \mathrm{H}_{10}\right)^{32}$ present a similar case, where the herringbone packing of pristine phenanthrene is completely absent from the products, whose structures are instead directed by the coordination of $\mathrm{Cs}^{+}$to the faces of the phenanthride anions with no incorporation of solvent moieties.

\section{Synthesis of $K_{2}$ Picene}


Synthesis of potassium-intercalated picene was carried out using the $\mathrm{KH}$ reaction protocols developed for pentacene, as direct reaction of picene with potassium metal produced similarly complex samples resulting from picene decomposition (Supplementary Figure 12). No reaction between picene and $\mathrm{KH}$ was observed below $180{ }^{\circ} \mathrm{C}$. Reaction outcome was highly sensitive to temperature and stoichiometry, with the nominal composition $\mathrm{K}_{2}$ Picene producing the highest quality samples, which are free of starting materials after two firings at $220^{\circ} \mathrm{C}$ (Figure $4 \mathrm{a}$ ). Any deviation from a nominal 2:1 KH:Picene ratio results in unconsumed starting material in the reaction product (Supplementary Figure 13). SXRD data from the products of reaction above $260{ }^{\circ} \mathrm{C}$ revealed an increase in the observed background and the appearance of a single Bragg peak in the PXRD patterns at low angle $\left(2 \theta_{\text {SXRD }} \approx 5.5^{\circ}\right)$ that could not be assigned to the new $\mathrm{K}_{2}$ Picene phase or to either of the starting materials (Figure $4 \mathrm{a}$ ). This decomposition product was also found in varying quantities in reactions carried out at lower temperatures but longer reaction times. Reaction times were therefore kept to the minimum required for all the KH to be consumed in the reaction whilst minimising decomposition of the K-intercalated product. The Raman and IR spectra of $\mathrm{K}_{2}$ Picene (Figure $4 \mathrm{~b}, \mathrm{c}$ ) are consistent with the presence of the piceneanion $^{33}$ (see Supplementary Sections 4 and 5, and Supplementary Figures 14-16), and a broad background feature in the Raman spectrum is consistent with the presence of a small amount of amorphous carbon. ${ }^{34}$ The presence of a fully intact picene molecule in $\mathrm{K}_{2}$ Picene is confirmed by re-oxidation in air at ambient temperature, which yields picene and potassium hydroxide as crystalline decomposition products (Supplementary Figures 17 and 18).

SXRD data (I11) collected from $\mathrm{K}_{2}$ Picene at ambient temperature were indexed to an orthorhombic unit cell of dimensions $a=7.6387(3) \AA, b=7.9342(3) \AA, c=25.9868(2) \AA$. No additional peak asymmetry or shoulders were observed in these data. The broad peak at $2 \theta_{\text {SXRD }}$ $\approx 5.5^{\circ}$ was excluded from the structure refinements. A structural model was produced by a similar protocol to that used for $\mathrm{K}_{2}$ Pentacene (see Methods and Supplementary Section 6), and Rietveld refinement of this model produced a good fit to the whole pattern $\left(R_{w p}=2.07 \%, R_{\exp }=\right.$ $0.28 \%$, Figure 5a, Supplementary Figure 19 and Supplementary Table 4) which compared well to the corresponding Le Bail fit $\left(R_{w p}=1.65 \%, R_{\exp }=0.28 \%\right.$, Supplementary Figure 20$)$.

\section{Structure of $\mathrm{K}_{2}$ Picene}

The structure of $\mathrm{K}_{2}$ Picene retains the herringbone motif and couples expansion of the original cell by $11.4 \%$ per picene with a molecular reorientation that increases $\omega$ from $57.89(7)^{\circ}$ to 88.4 $(2)^{\circ}$ to create pockets within the layers for the inserted cations (Figure 5b). The refined C-C bond distance of the picene dianion of 1.437(7) $\AA$ is longer than the mean C-C distance of neutral picene $(1.40(3) \AA ̊)$. Unlike $\mathrm{K}_{2}$ Pentacene, the asymmetric unit contains a single picene molecule, and there is a single pocket of approximately square cross-section located within the layers. The pocket contains two potassium cations, each disordered around a maximum of scattering density at either end of the pocket, producing three partially occupied sites (K1, K2 and K3, Figure 5c) whose occupancy-weighted mean position is shown in Figure 5d. These three sites have refined occupancies of $0.153(3), 0.504(4)$ and $0.355(4)$ respectively and are spread over $2.70(3) \AA$ A hence locally only one of these sites may be occupied by potassium. The site occupied locally by potassium at one end of the pocket must also affect the site occupied at the 
opposite end of the pocket: for example, within a single pocket the two K3 sites are separated by 2.74(1) $\AA$, which is too short for simultaneous local occupancy.

The molecular reorientation that creates the pockets strengthens the $\mathrm{C}-\mathrm{H} . . . \pi$ interactions in comparison with the picene parent structure by positioning the hydrogens along the long edges of the molecule approximately perpendicular to and close to the midpoint between the long edges of the neighbouring aromatic systems: the mean centroid offset $\delta$ decreases from $0.70(2) \AA$ in picene to $0.10(1) \AA$, and the mean C-H...aromatic plane distance, $d$, shortens from 2.75(7) $\AA$ to 2.65(18) Å despite the structural expansion upon cation insertion (see Figures 6c and 6d, Supplementary Figures 21 and 22, and extended structure comparison in Supplementary Section 7).

The point symmetry of a picene molecule is $\mathrm{C}_{2 \mathrm{v}}(\mathrm{mm} 2)$ and consequently the molecular orientation has an additional degree of rotational freedom compared to pentacene $\left(\mathrm{D}_{2 \mathrm{~h}}, \mathrm{mmm}\right)$. In $\mathrm{K}_{2}$ Picene the molecular anions have a disordered distribution of two orientations (related by a rotation of $180^{\circ}$ about the long axis of the molecule, see Figure 5c and Supplementary Figure 23). This orientational disorder of the picene anions produces a complex range of potential local coordination environments: in a single pocket defined by four picene anions within the layer, which may each adopt one of two orientations, there are 16 possible configurations. The impact of this anion orientational disorder on the potassium-accessible volume of the pockets was investigated computationally by constructing several $2 \times 2 \times 1$ supercells based on the refined crystal structure, then randomising the orientation of the anions in each supercell prior to calculation of the available void space (see Supplementary Section 8). This produced a distribution of pocket types whose available void space is influenced strongly by the local anion configuration (Figure $6 \mathrm{~g}$ and Supplementary Figure 24). In contrast, a picene array with a single molecular orientation (Figure $5 \mathrm{~d}$ ) produces a uniform pocket type (Figure 6e, f). The positional disorder of the potassium cations in the refined crystal structure is therefore consistent with the anion disorder and the resulting distribution of potential sites. Figure 6a shows one set of orientations that correspond to local occupation of both of the K2 sites, where the closest K...C contacts are to two carbons at the extrema of each of the four picene molecules that define the pocket. As with $\mathrm{K}_{2}$ Pentacene, $\eta^{6}$ coordination of potassium by the picene dianion is not possible in $\mathrm{K}_{2}$ Picene, as these sites are obstructed by $\mathrm{C}-\mathrm{H} \ldots \pi$ interactions between neighbouring molecules (Supplementary Figure 22). The resulting K...C contacts lie in the range 2.757(9) to 3.348(8) A (Supplementary Table 5), and inspection of the available void space indicates that there are several cation locations where physically reasonable $\mathrm{K}-\mathrm{C}$ distances can be achieved with the distribution of anion orientations (Figure 6g). Some configurations exist with large enough void space at the centre of the pocket to accommodate additional cations (Figure 6e - g), which are not occupied in $\mathrm{K}_{2}$ Picene. It is conceivable that this central position could be occupied if the other two $\mathrm{K}^{+}$ions were at the extreme ends of the pocket (i.e., at or near the $\mathrm{K} 1$ sites, which are separated by 8.01(3) Å, Figure 5c). Such an arrangement would produce a structure similar to that predicted for $\mathrm{K}_{3}$ Picene. ${ }^{35}$

\section{Electronic Structure}


Infra-red and magnetisation measurements show that the charge transferred from the two potassium cations per pocket in both $\mathrm{K}_{2}$ Picene and $\mathrm{K}_{2}$ Pentacene affords insulators. The IR absorption spectra provide no evidence for conventional metallic behaviour ${ }^{36}$ in either compound, as indicated by the low background and the absence of the characteristic Fano line shape (Figures 2c, 4c and Supplementary Figure 16). Magnetisation measurements of $\mathrm{K}_{2}$ Pentacene and $\mathrm{K}_{2}$ Picene showed that both compounds exhibit weak, temperature-dependent paramagnetic signals (see insets of Figures $2 \mathrm{a}$ and $4 \mathrm{a}$, respectively). A modified Curie-Weiss fit to these data (see Methods) yields a molar Curie constant $\left(C_{m o l}\right)$ of $2.9(1) \times 10^{-3} \mathrm{~cm}^{3} \mathrm{~K} \mathrm{~mol}^{-1}$ for $\mathrm{K}_{2}$ Pentacene, corresponding to a value of $0.77(3) \%$ impurity spins; a value of $C_{m o l}=1.38(3) \times$ $10^{-2} \mathrm{~cm}^{3} \mathrm{~K} \mathrm{~mol}^{-1}$ was similarly determined for $\mathrm{K}_{2}$ Picene, corresponding to $3.70(6) \%$ impurity spins. As neither compound is metallic, these small magnetisation values indicate the formation of a singlet ground state: this is consistent for $\mathrm{K}_{2}$ Pentacene with filling of the LUMO-derived band because of the separation of over $1 \mathrm{eV}$ between LUMO and LUMO+1 in bulk pentacene ${ }^{37}$, consistent with the conclusions of recent magnetic and spectroscopic studies of $\mathrm{K}$ intercalated polyacenes. ${ }^{19}$ Density functional theory (DFT) calculations show that the first unoccupied electronic band of solid pristine picene is derived from both the LUMO and LUMO+1 orbitals of the two picene molecules in the cell. ${ }^{35}, 38$ In a rigid band treatment, charging the two picene molecules to dianions would place four electrons into this band, leaving it half filled (Supplementary Figure 25) ${ }^{38}$, suggesting that $\mathrm{K}_{2}$ Picene should be metallic. However DFT calculations on the experimentally determined $\mathrm{K}_{2}$ Picene structure here show that the fully occupied band deriving from the LUMO orbitals is separated from an unoccupied band deriving from the LUMO+1 orbitals (Supplementary Figure 26). A similar separation of LUMO and LUMO+1-derived bands is found in DFT calculations on one computationally proposed $\mathrm{K}_{3}$ Picene structure ${ }^{35}$, although the bands retain mixed LUMO and LUMO+1 character in another variant 38 . $\mathrm{K}_{2}$ Picene is thus predicted by DFT to be a band insulator with a gap in the region of $0.2 \mathrm{eV}$. Removing the $\mathrm{K}^{+}$ions from the crystal structure of $\mathrm{K}_{2}$ Picene does not cause the LUMO and LUM0+1 derived bands to recombine (Supplementary Figure 27), suggesting that there is a structural contribution to the splitting of the LUMO and LUMO+1 bands associated with extensive rearrangement of the herringbone packing. The change in the computed geometry of picene in $\mathrm{K}_{2}$ Picene compared to solid picene is consistent with the occupation of the LUMO orbital when forming the dianion (Supplementary Figure 28). Density functional plus dynamical mean field theory (DFT+DMFT) calculations on $\mathrm{K}_{\mathrm{x}} \mathrm{Picene}^{39}$ suggest the opening of a Mott gap by electron correlation in both LUMO and LUMO+1 bands in a multi-orbital correlated model, ${ }^{40}$ which is required to explain the insulating behaviour of $\mathrm{Cs}_{2}$ Phenanthrene, ${ }^{32}$ and such correlation effects, which are not captured at the level of theory used here, may also contribute to the gap in the picene dianion solid. Raman spectroscopy does identify differences in the electronic behaviour of the $\mathrm{K}_{2}$ Pentacene and $\mathrm{K}_{2}$ Picene: high-frequency Raman modes display significant broadening in $\mathrm{K}_{2}$ Picene, implying electron-phonon coupling, which is not observed in $\mathrm{K}_{2}$ Pentacene (see Supplementary Section 4). Magnetisation measurements in a field of $100 \mathrm{Oe}$ show both $\mathrm{K}_{2}$ Pentacene and $\mathrm{K}_{2}$ Picene are non-superconducting above $2 \mathrm{~K}$ (Supplementary Figure 29).

\section{Conclusion}


The formation of the expanded herringbone structures of $\mathrm{K}_{2}$ Pentacene and $\mathrm{K}_{2}$ Picene is driven by the generation of sites with the correct chemistry to accommodate the potassium cations. The void space in the parent herringbone structures lies between the PAH layers and is lined with $\mathrm{C}-\mathrm{H} \sigma$ bonds, whereas the electron transfer from potassium to PAH that drives cation insertion will necessarily place the negative charge in the molecular $\pi$ systems. Structural rearrangement is thus needed to allow the potassium cations access to the aromatic rings, in contrast to both $\mathrm{C}_{60}$ and graphite, where such sites are immediately apparent on inspection of the host structure and occupied upon intercalation. Reorientation of the (phen)acenes opens pockets within the layers that are lined by the $\pi$ systems and contain multiple cation sites that are occupied in a manner controlled by the precise (phen)acene molecular geometry and the C$\mathrm{H} . . . \pi$ contacts within the structure: the strengthening of these supramolecular interactions, absent in carbon-only hosts, compensates for the loss in global van der Waals-type interaction expected upon the expansion of the hydrocarbon arrays associated with intercalation. The inserted cations thus have to produce their own sites in the product structure. The barrier to this reorganisation may require reaction conditions sufficiently harsh that molecular decomposition competes with insertion unless the redox properties of the reducing agent are carefully controlled. The observation of extensive PAH decomposition on direct high temperature reaction with potassium metal suggests that a search for an explanation of the reported superconductivity in $\mathrm{K}_{x}$ Picene and other alkali metal PAH reaction products should include understanding the possible chemical modifications to these molecules under the reaction conditions required for potassium insertion. Control of the relationship between cation size, charge and chemistry and the potential restructuring is needed to access the different cation:anion ratios and $\mathrm{PAH}$ charge states, but this requires development of chemistry to favour the nucleation of the necessary molecular reorientation over competing molecular decomposition. This will allow designed modification of electronic structure by band filling and band overlap of the LUMO and LUMO+1 levels, giving access to states such as multi-orbital correlated insulators $^{32}$. Redox-controlled solid-state synthetic routes to solvent-free alkali metal $\mathrm{PAH}$ salts, together with complementary mild solution-based synthetic routes ${ }^{32}$ that offer alternative reaction pathways that are not influenced at each stage by the supramolecular interactions between the PAHs themselves, will play a key role in realising the scope of the synthetic and associated functional opportunity.

\section{Methods:}

Synthesis. All reagents and samples were stored and handled in an argon-filled glove box $\mathrm{CO}_{2}$ and $\mathrm{H}_{2} \mathrm{O}<1 \mathrm{ppm}$ ). All reactions were carried out in Pyrex ampoules with an internal diameter of $6 \mathrm{~mm}$ and an approximate length of $10 \mathrm{~cm}$ and sealed under vacuum $\left(<10^{-4} \mathrm{mbar}\right)$. After trial reactions with potassium metal (see Supplementary Section 1), KH was selected as a milder reducing agent. Reactions between pentacene/picene (TCI Chemicals, sublimed grade $\geq 99.9 \%$ ) and KH (Sigma Aldrich, washed with anhydrous pentane under inert atmosphere) were prepared by grinding $50 \mathrm{mg}$ of the (phen)acene $(0.180 \mathrm{mmol})$ with $14.4 \mathrm{mg} \mathrm{KH}(0.359 \mathrm{mmol}, 2$ equivalents) using an agate mortar and pestle. The resulting powders were pressed into $5 \mathrm{~mm}$ diameter pellets under 2 tonnes of force before heating in Pyrex ampoules sealed under 
vacuum. The highest quality samples of $\mathrm{K}_{2}$ Pentacene and $\mathrm{K}_{2}$ Picene were afforded by reaction at $280{ }^{\circ} \mathrm{C}$ for two periods of five days, and $220{ }^{\circ} \mathrm{C}$ for two periods of two days; respectively; with intermediate regrinding and pelletising between each heating period.

Powder X-ray diffraction. PXRD data were collected using a Bruker-AXS D8 Advance

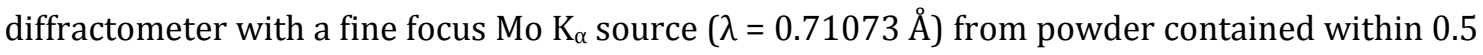
$\mathrm{mm}$ diameter borosilicate capillaries. Synchrotron X-ray powder diffraction (SXRD) data were collected at room temperature from powder contained within $0.5 \mathrm{~mm}$ diameter borosilicate capillaries using the I11 beam line at Diamond Light Source $(\lambda=0.825685(1) \AA$ ); a data set was also collected at $100 \mathrm{~K}$ from a sample of $\mathrm{K}_{2}$ Pentacene. The initial Rietveld refinements were performed using GSAS, 41,42 and the final round of Rietveld refinements were performed using TOPAS Academic version $5 .{ }^{43} \mathrm{Ab}$-initio structure solution from the powder diffraction data was conducted by simulated annealing in FOX ${ }^{44}$ with symmetry identification using FINDSYM ${ }^{45}$, followed by an iterative series of Rietveld refinements with the acene molecules modelled as rigid bodies with a single refinable C-C distance and fixed C-C-C angles. A detailed account of these structure solution routes is provided in Sections 2 and 6 of the Supplementary Information.

Raman and IR spectroscopy. Raman spectra of pentacene-based materials were collected using a Renishaw inVia Raman Spectrometer using an excitation wavelength of $785 \mathrm{~nm}$ and a power of $50 \mu \mathrm{W}$ from samples contained within silica capillaries. Raman spectra from picenebased materials were obtained using a laser with an excitation wavelength of $633 \mathrm{~nm}$ and a power of $370 \mu \mathrm{W}$. The spectra were collected in backscattering geometry by a confocal microscope attached to a Horiba Jobin-Yvon LabRAM HR spectrometer. Infrared spectra were obtained from samples pressed into $\mathrm{KBr}$ pellets using either a Bruker Alpha FT-IR spectrometer (pentacene-based materials) or Perkin Elmer Spectrum 100 FTIR spectrometer (picene-based materials). Further details can be found in Sections 4 and 5 of the Supplementary Information.

Magnetometry. SQUID magnetometry data were collected from samples contained within silica ampoules using a Quantum Design MPMS XL-7 SQUID magnetometer. Magnetic susceptibility was determined from measurement of the difference in magnetisation in $5 \mathrm{~T}$ and $3 \mathrm{~T}$ fields over the temperature range $10-300 \mathrm{~K}$, corrected for the core diamagnetism of $\mathrm{K}^{+}$and of pentacene/picene in addition to the aromatic rings. A modified Curie Weiss Law of the form $\chi=$ $(C /(T-\theta))+K$ yielded values of $C=0.0029(1) \mathrm{cm}^{3} \mathrm{~K} \mathrm{~mol}^{-1}, \theta=-1.0(6) \mathrm{K}, K=4.9(1) \times 10^{-5} \mathrm{~cm}^{3} \mathrm{~mol}^{-}$ ${ }^{1}$ in $\mathrm{K}_{2}$ Pentacene and $C=0.0138(3) \mathrm{cm}^{3} \mathrm{~K} \mathrm{~mol}^{-1}, \theta=-5.5(4) \mathrm{K}, K=4.73(2) \times 10^{-4} \mathrm{~cm}^{3} \mathrm{~mol}^{-1}$ $\mathrm{K}_{2}$ Picene. Further details are available in Section 9 of the Supplementary Information.

ICP-OES measurements. Measurements were performed utilising a Spectro Ciros ICP-OES radial view instrument. Samples were dissolved in dilute nitric acid. The compositions determined were $22.4 \mathrm{wt} . \%$ and $22.9 \mathrm{wt} . \% \mathrm{~K}$ for $\mathrm{K}_{2}$ Pentacene and $\mathrm{K}_{2}$ Picene, respectively; consistent with the theoretical value of $21.9 \%$. Further details of the ICP-OES measurements are given in Section 10 of the Supplementary Information.

Computation. Plane-wave based density functional theory (DFT) calculations were performed using the VASP programme ${ }^{46}$. The optB86b-vdW functional ${ }^{47}$ was used to improve the description of van der Waals interactions over other semi-local DFT functionals, with a plane- 
wave cutoff energy of $520 \mathrm{eV}$. Core electrons were treated using the projector augmented-wave method ${ }^{48}$. Unit cell parameters and atomic positions were relaxed until all forces were reduced to below $10^{-3} \mathrm{eV} / \AA$. Calculations on $\mathrm{K}_{2}$ Picene were performed using an ordered unit cell with $\mathrm{P} 2{ }_{1} 2_{1} 2_{1}$ symmetry, corresponding to a particular choice of the disordered picene molecules in the refined crystal structure as shown in Supplementary Figure 33, with a $7 \times 7 \times 2 k$-point grid. Calculations on pristine solid picene were performed using the reported experimental structure ${ }^{13}$ as a starting point for geometry relaxation, and a $6 \times 9 \times 4 k$-point grid. $k$-point strings used to plot band structures were generated using the AFLOW framework ${ }^{49}$. Projections of the density of states onto the molecular orbitals of individual picene molecules was performed using a modified version of VASP and related external routines ${ }^{50}$.

CCDC 1494829-1494830 contain the supplementary crystallographic data for this paper. These data can be obtained free of charge from The Cambridge Crystallographic Data Centre via www.ccdc.cam.ac.uk/data request/cif. Original data is available at the University of Liverpool's DataCat repository at http://datacat.liv.ac.uk/.

\section{Acknowledgments}

We acknowledge financial support by the UK Engineering and Physical Sciences Research Council (EP/K027255 and EP/K027212), the European Union/JST SICORP-LEMSUPER FP7NMP-2011-EU-Japan project (contract no. NMP3-SL-2011-283214), the Mitsubishi Foundation, the JSPS under the Scientific Research on Innovative Areas "J-Physics" Project (No 15H05882), the "World Premier International (WPI) Research Center Initiative for Atoms, Molecules and Materials," Ministry of Education, Culture, Sports, Science, and Technology of Japan and the Japan Science and Technology Agency under the ERATO Isobe Degenerate $\pi$-Integration Project. We thank the Diamond Light Source for access to synchrotron X-ray facilities, and C.C. Tang, C.A. Murray and P. Adamson for beamline assistance. We thank Prof. Mats Persson for use of his XVASP code and related external routines for projecting density of states onto molecular orbitals. We thank the Royal Society for a Newton International Fellowship (G.K.). MJR is a Royal Society Research Professor.

\section{Author Contributions}

K.P. and M.J.R. conceived and designed the project. M.J.R. directed and coordinated the research. F.D.R carried out initial syntheses using KH that identified both phases, and performed initial structure determination of both phases. M.J.P. completed the structural analyses with C.I.H and C.C. C.I.H performed final syntheses and, with M.J.P., finalised the link between synthesis and structure. G.F.S.W defined the structural evolution of the intermolecular interactions. S.K. and A.Y.G. developed early reaction protocols, and R. H. C. undertook early structural work. D.A. computed the evolution of the void space within the material that connects the molecular packing to the cation distribution. M.S.D performed and interpreted electronic structure 
calculations. G.K. performed and interpreted Raman and infra-red measurements. F.D.R. and M.J.R. wrote the first draft of the paper, which was then completed with input from all authors. 
Figure 1. Available void space in carbon-based molecular crystal structures. (a) the herringbone structure of picene: 13 the red surface represents voids at least $2.5 \AA$ from an atomic site, showing that the largest available sites are non-adjacent to the $\pi$-system. (b) the face centred cubic structure of $\mathrm{C}_{60}:{ }^{15}$ green spheres highlight each $\mathrm{C}_{60}$ molecule and the red surface represents voids at least $2.65 \AA$ from an atomic site, revealing the larger octahedral and smaller tetrahedral sites (note that the body-centred packings of $\mathrm{K}_{4} \mathrm{C}_{60} 51$ and $\mathrm{K}_{6} \mathrm{C}_{60} 52$ are related to the body-centred tetragonal representation of fcc pristine $\mathrm{C}_{60}$ ). These sites are both larger than those in picene, and are adjacent to the $\pi$-system.

Figure 2: Characterisation data for $K_{2}$ Pentacene. (a) Room temperature SXRD data collected from pentacene (blue) and $\mathrm{K}_{2}$ Pentacene (red). Inset: Magnetisation data as a function of temperature collected from $\mathrm{K}_{2}$ Pentacene (points), with a modified Curie-Weiss law fit (line). (b) Raman and (c) infrared spectra collected from pentacene and $\mathrm{K}_{2}$ Pentacene.

Figure 3: Crystal structure of $\mathbf{K}_{2}$ Pentacene. (a) Single-phase Rietveld refinement against SXRD data collected from $\mathrm{K}_{2}$ Pentacene at $100 \mathrm{~K}(\lambda=0.825685(2) \AA)$, with black observed data points, the calculated pattern (red) and difference plot (green) respectively. Insets show misfit to the 002 reflection (left) and fit to high angle region (right). (b) Two-phase fit to the same data with a Rietveld refinement of the majority phase (dashed orange) and a Le Bail refinement of the secondary phase (dashed blue). Insets show improved fit to the 002 reflection and the high angle region, see Supplementary Table 1 for agreement factors and refined lattice parameters. (c) Reorientation of the herringbone array of molecular species found in pristine pentacene, ${ }^{16}$ which creates $\mathrm{K}$ sites in $\mathrm{K}_{2}$ Pentacene that are close to the aromatic $\pi$-systems and strengthens $\mathrm{C}-\mathrm{H} \ldots \pi$ contacts. The black dashed lines denote the molecular orientations with intermolecular angles labelled. (d) The final structural model of $\mathrm{K}_{2}$ Pentacene from two-phase refinement against SXRD data containing four half-occupied $\mathrm{K}$ sites in pockets bounded by four pentacene anions. Further structural details are available in Supplementary Information. (e) Available void space (representing K-C distances of $2.7-2.9 \AA$ ) in the $\mathrm{K}_{2}$ Pentacene structure. The observed K sites correspond to the largest spaces within the pockets in the pentacene array, as indicated by the dotted lines connecting (d) and (e).

Figure 4: Characterisation data for $\mathrm{K}_{2}$ Picene. (a) SXRD data collected from sublimed picene (blue), $\mathrm{K}_{2}$ Picene (red, observed count rate offset by 1 arbitrary unit for clarity) and partially decomposed $\mathrm{K}_{2}$ Picene yielded by the reaction of $2 \mathrm{KH}+$ Picene at $260^{\circ} \mathrm{C}$ (green, observed count rate multiplied by a factor of 30 for clarity). Dashed line at $2 \theta=5.5^{\circ}$ highlights diffraction peak observed due to decomposition of $\mathrm{K}_{2}$ Picene. Inset: Magnetisation as a function of temperature collected from $\mathrm{K}_{2}$ Picene (black circles), with a modified Curie-Weiss law fit (red line). (b) Raman and (c) infrared spectra collected from picene and $\mathrm{K}_{2}$ Picene. The shift of the most intense Raman peak of pristine picene peaks indicates the presence of the picene ${ }^{2-}$ anion. ${ }^{33}$

Figure 5: Crystal structure of $\mathbf{K}_{2}$ Picene. (a) Rietveld refinement of the $\mathrm{K}_{2}$ Picene structure $\left(R_{\mathrm{wp}}=2.07 \%, R_{\exp }=0.28\right.$ $\%$ Pbca, $a=7.6387(3) \AA, b=7.9342(3) \AA, c=25.9868(2) \AA$ ) against data collected from a sample at $100 \mathrm{~K}$ using the I11 beamline $(\lambda=0.825685(2) \AA)$, with black circles representing observed points, and red and green lines showing calculated and difference plots; respectively. The region between $5.25^{\circ}<\theta<5.75^{\circ}$ was excluded due to an impurity peak. (b) The reorientation of the picene molecular anions away from their positions in pristine picene ${ }^{13}$ creates sites for potassium, with the anions arranged at $88.45(2)^{\circ}$ to each other. Dashed black lines denote the molecular orientations. (c) The final refined model of the structure containing a superimposition of the two picene orientations 
(green and blue) with partially-occupied potassium sites shown in red. (d) Simplified structure of $\mathrm{K}_{2}$ Picene with a single picene orientation and the (occupancy-weighted) average K site (orange). Hydrogen atoms omitted for clarity.

Figure 6: Local structure in $K_{2}$ Picene. (a) A single pocket with the $\mathrm{K} 2$ sites occupied (solid red spheres), surrounded by picene anions with orientations generating physical K2-C distances. Semi-transparent spheres show unoccupied K1 and K3 sites. K-C bond distances between distinct K2 sites (labelled A and B), and molecules (U-X, coloured green or blue depending on orientation) available in Supplementary Table 5. (b) A single K2 site with K-C contacts arising from the same array of molecular orientations. (c) The molecular orientation of neighbouring picene moieties in picene and (d) in $\mathrm{K}_{2}$ Picene, viewed down the molecular long axis of, and perpendicular to, the picene acting as acceptor in the $\mathrm{C}-\mathrm{H}$... $\pi$ interaction (shown in green). The donor molecule shown in red. The $\mathrm{C}-\mathrm{H}$ bonds in the donor approach the acceptor closer to perpendicular and to the aromatic ring centroids in $\mathrm{K}_{2}$ Picene than in picene. (e, f) Available void spaces from the picene array in $\mathrm{K}_{2}$ Picene with a single picene molecular orientation, viewed down the [001] direction (e), and [100] direction (f). (g) Void space from the picene array in $\mathrm{K}_{2}$ Picene with a disordered arrangement of picene molecules, viewed down the (001) direction. Contours show regions $\mathrm{K}$ can occupy with a $d_{\mathrm{K}-\mathrm{C}}$ of $2.8-3.0 \AA$. 


\section{References}

1. Fleming R. M. et al. Relation of structure and superconducting transition temperatures in $\mathrm{A}_{3} \mathrm{C}_{60}$. Nature, 352, 787-788 (1991).

2. Hebard A. F. et al. Superconductivity at $18 \mathrm{~K}$ in potassium doped $\mathrm{C}_{60}$. Nature, 350, 600601 (1991).

3. Tanigaki K. et al. Superconductivity at $33 \mathrm{~K}$ in $\mathrm{Cs}_{\mathrm{x}} \mathrm{Rb}_{\mathrm{y}} \mathrm{C}_{60}$. Nature, 352, 222-223 (1991).

4. Ekimov E. A. et al. Superconductivity in diamond. Nature, 428, 542-545 (2004).

5. Weller T. E., Ellerby M., Saxena S. S., Smith R. P. \& Skipper N. T. Superconductivity in the intercalated graphite compounds $\mathrm{C}_{6} \mathrm{Yb}$ and $\mathrm{C}_{6} \mathrm{Ca}$. Nat. Phys., 1, 39-41 (2005).

6. Ganin A. Y. et al. Bulk superconductivity at $38 \mathrm{~K}$ in a molecular system. Nat. Mater., 7, 367-371 (2008).

7. Stephens P. W. et al. Structure of single phase superconducting $\mathrm{K}_{3} \mathrm{C}_{60}$. Nature, 351, 632634 (1991).

8. Mitsuhashi R. et al. Superconductivity in alkali-metal-doped picene. Nature, 464, 76-79 (2010).

9. Wang X. F. et al. Superconductivity at $5 \mathrm{~K}$ in alkali-metal-doped phenanthrene. Nat. Commun., 2, 507 (2011).

10. Kubozono Y. et al. Metal-intercalated aromatic hydrocarbons: a new class of carbonbased superconductors. Phys. Chem. Chem. Phys., 13, 16476-16493 (2011).

11. Xue M. et al. Superconductivity above $30 \mathrm{~K}$ in alkali-metal-doped hydrocarbon. Sci. Rep., 2, 389 (2012).

12. Trotter J. Crystal and molecular structure of phenanthrene. Acta Crystallogr., 16, 605608 (1963).

13. De A., Ghosh R., Roychowdhury S. \& Roychowdhury P. Structural analysis of picene, $\mathrm{C}_{22} \mathrm{H}_{14}$. Acta Crystallogr. Sect. C: Cryst. Struct. Commun., 41, 907-909 (1985). 
14. Kaefer D., El Helou M., Gemel C. \& Witte G. Packing of planar organic molecules: Interplay of van der waals and electrostatic interaction. Cryst. Growth Des., 8, 3053-3057 (2008).

15. Burgi H. B., Restori R. \& Schwarzenbach D. Structure of $\mathrm{C}_{60}$ - Partial orientational order in the room-temperature modification of $\mathrm{C}_{60}$. Acta Crystallogr. Sect. B: Struct. Sci., 49, 832-838 (1993).

16. Desiraju G. R. \& Gavezzotti A. Crystal structures of polynuclear aromatic hydrocarbons classification, rationalization and prediction from molecular structure. Acta Crystallogr. Sect. B: Struct. Sci., 45, 473-482 (1989).

17. Mori T. \& Ikehata S. ESR study on potassium doped pentacene. Solid State Commun., 101, 213-218 (1997).

18. Phan Q. T. N., Heguri S., Tanabe Y., Shimotani H. \& Tanigaki K. Systematic Study of the Electronic States in Electron-Doped Polyacenes. Eur. J. Inorg. Chem., 4033-4038 (2014).

19. Phan Q. T. N., Heguri S., Tamura H., Nakano T., Nozue Y. \& Tanigaki K. Two different ground states in K-intercalated polyacenes. Phys. Rev. B, 93, 075130 (2016).

20. Nanditha D. M., Dissanayake M., Adikaari A. A. D. T., Curry R. J., Hatton R. A. \& Silva S. R. P. Nanoimprinted large area heterojunction pentacene- $\mathrm{C}_{60}$ photovoltaic device. Appl. Phys. Lett., 90, 253502 (2007).

21. Koch N. Organic electronic devices and their functional interfaces. ChemPhysChem, 8, 1438-1455 (2007).

22. Yamashita Y. Organic semiconductors for organic field-effect transistors. Sci. Technol. Adv. Mater., 10, 024313 (2009).

23. Craciun M. F., Giovannetti G., Rogge S., Brocks G., Morpurgo A. F. \& van den Brink J. Evidence for the formation of a Mott state in potassium-intercalated pentacene. Phys. Rev. B, 79, 125116 (2009).

24. Minakata T., Ozaki M. \& Imai H. Conducting thin films of pentacene doped with alkaline metals. J. Appl. Phys., 74, 1079-1082 (1993).

25. Vanýsek J. CRC Handbook of Chemistry and Physics (2011). 
26. Bergman I. The polarography of polycyclic aromatic hydrocarbons and the relationship between their half-wave potentials and absorption spectra. Trans. Faraday Society, 50, 829-838 (1954).

27. Szczepanski J., Wehlburg C. \& Vala M. Vibrational and electronic spectra of matrix isolated pentacene cations and anions. Chem. Phys. Lett., 232, 221-228 (1995).

28. Mattheus C. C., Dros A. B., Baas J., Meetsma A., de Boer J. L. \& Palstra T. T. M. Polymorphism in pentacene. Acta Crystallogr. Sect. C: Cryst. Struct. Commun., 57, 939941 (2001).

29. Desiraju G. R. \& Steiner T. The Weak Hydrogen Bond In Structural Chemistry and Biology. Oxford Science Publications (1999).

30. Scott T. A. et al. After 118 years, the isolation of two common radical anion reductants as simple, stable solids. Chem. Commun., 65-67 (2009).

31. Bock H., Gharagozloo-Hubmann K., Sievert M., Prisner T. \& Havlas Z. Single crystals of an ionic anthracene aggregate with a triplet ground state. Nature, 404, 267-269 (2000).

32. Takabayashi Y. et al. $\pi$-electron $S=1 / 2$ quantum spin liquid state in an ionic polyaromatic hydrocarbon. (accompanying paper).

33. Kambe T. et al. Synthesis and physical properties of metal-doped picene solids. Phys. Rev. $B, 86,214507$ (2012).

34. Ferrari A. C. \& Robertson J. Raman spectroscopy of amorphous, nanostructured, diamond-like carbon, and nanodiamond. Philos. Trans. R. Soc. London, Ser. A, 362, 24772512 (2004).

35. Naghavi S. S. \& Tosatti E. Crystal structure search and electronic properties of alkalidoped phenanthrene and picene. Phys. Rev. B, 90, 075143 (2014).

36. Kamaras K. \& Klupp G. Metallicity in fullerides. Dalton Trans., 43, 7366-7378 (2014).

37. Claudia A.-D., Dmitrii N., Peter P. \& Christian M. The role of polymorphism in organic thin films: oligoacenes investigated from first principles. New J. Phys., 11, 125010 (2009).

38. Kosugi T., Miyake T., Ishibashi S., Arita R. \& Aoki H. First-Principles Electronic Structure of Solid Picene. J. Phys. Soc. Jpn., 78, 113704 (2009). 
39. Ruff A. et al. Absence of Metallicity in K-doped Picene: Importance of Electronic Correlations. Phys. Rev. Lett., 110, 216403 (2013).

40. Werner P. \& Millis A. J. High-Spin to Low-Spin and Orbital Polarization Transitions in Multiorbital Mott Systems. Phys. Rev. Lett., 99, 126405 (2007).

41. Larson A. C. \& von Dreele R. General Structure Analysis System (GSAS). Los Alamos National Laboratory Report, 86-748 (1994).

42. Toby B. H. EXPGUI, a graphical user interface for GSAS. J. Appl. Crystallogr., 34, 210-213 (2001).

43. Coelho A. A. TOPAS-Academic v5. Coelho Software. (2012)

44. Favre-Nicolin V. \& Cerny R. FOX, 'free objects for crystallography': a modular approach to ab initio structure determination from powder diffraction. J. Appl. Crystallogr., 35, 734-743 (2002).

45. Stokes H. T. \& Hatch D. M. FINDSYM: program for identifying the space-group symmetry of a crystal. J. Appl. Crystallogr., 38, 237-238 (2005).

46. Kresse G. \& Furthmuller J. Efficient iterative schemes for ab initio total-energy calculations using a plane-wave basis set. Phys. Rev. B, 54, 11169-11186 (1996).

47. Klimes J., Bowler D. R. \& Michaelides A. Van der Waals density functionals applied to solids. Phys. Rev. B, 83, 195131 (2011).

48. Kresse G. \& Joubert D. From ultrasoft pseudopotentials to the projector augmentedwave method. Phys. Rev. B, 59, 1758-1775 (1999).

49. Curtarolo S. et al. AFLOW: An automatic framework for high-throughput materials discovery. Comput. Mater. Sci., 58, 218-226 (2012).

50. Lorente N. \& Persson M. Theoretical aspects of tunneling-current-induced bond excitation and breaking at surfaces. Faraday Discuss., 117, 277-290 (2000).

51. Fleming R. M. et al. Preparation and structure of the alkali-metal fulleride $\mathrm{A}_{4} \mathrm{C}_{60}$. Nature, 352, 701-703 (1991). 
52. Zhou 0. et al. Structure and bonding in alkali-metal-doped $\mathrm{C}_{60}$. Nature, 351, 462-464 (1991). 


\section{Picene}
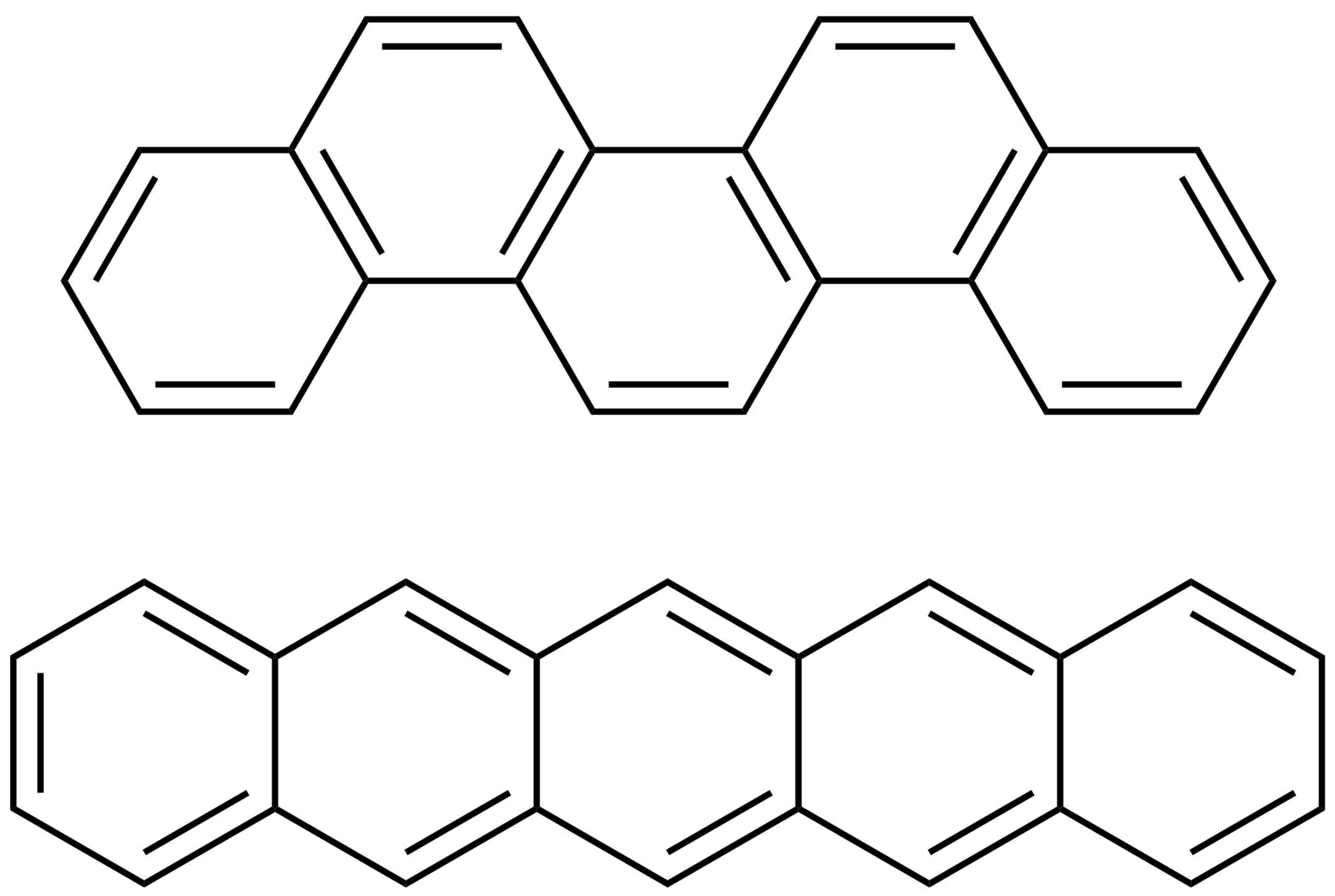

Pentacene 


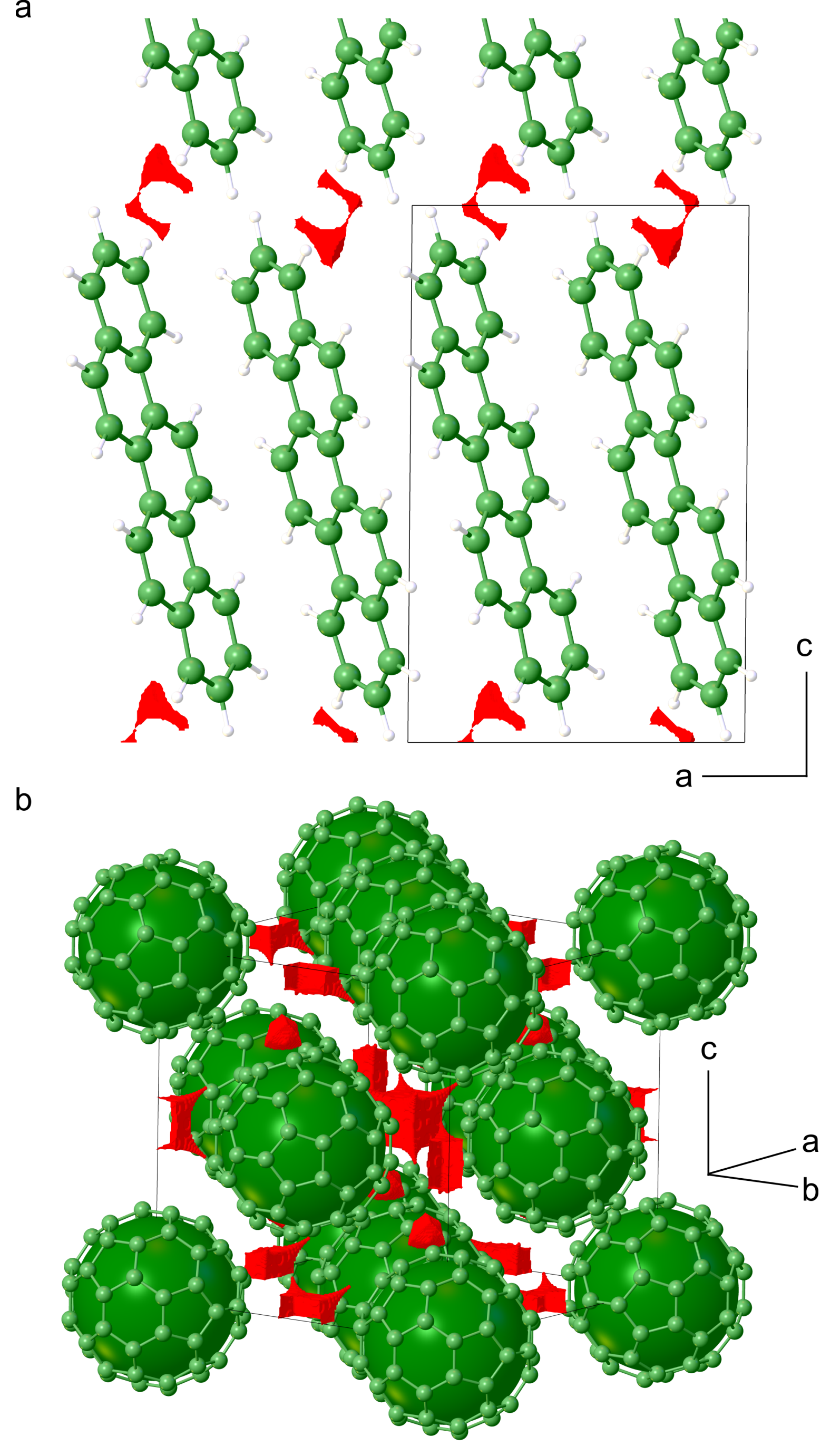



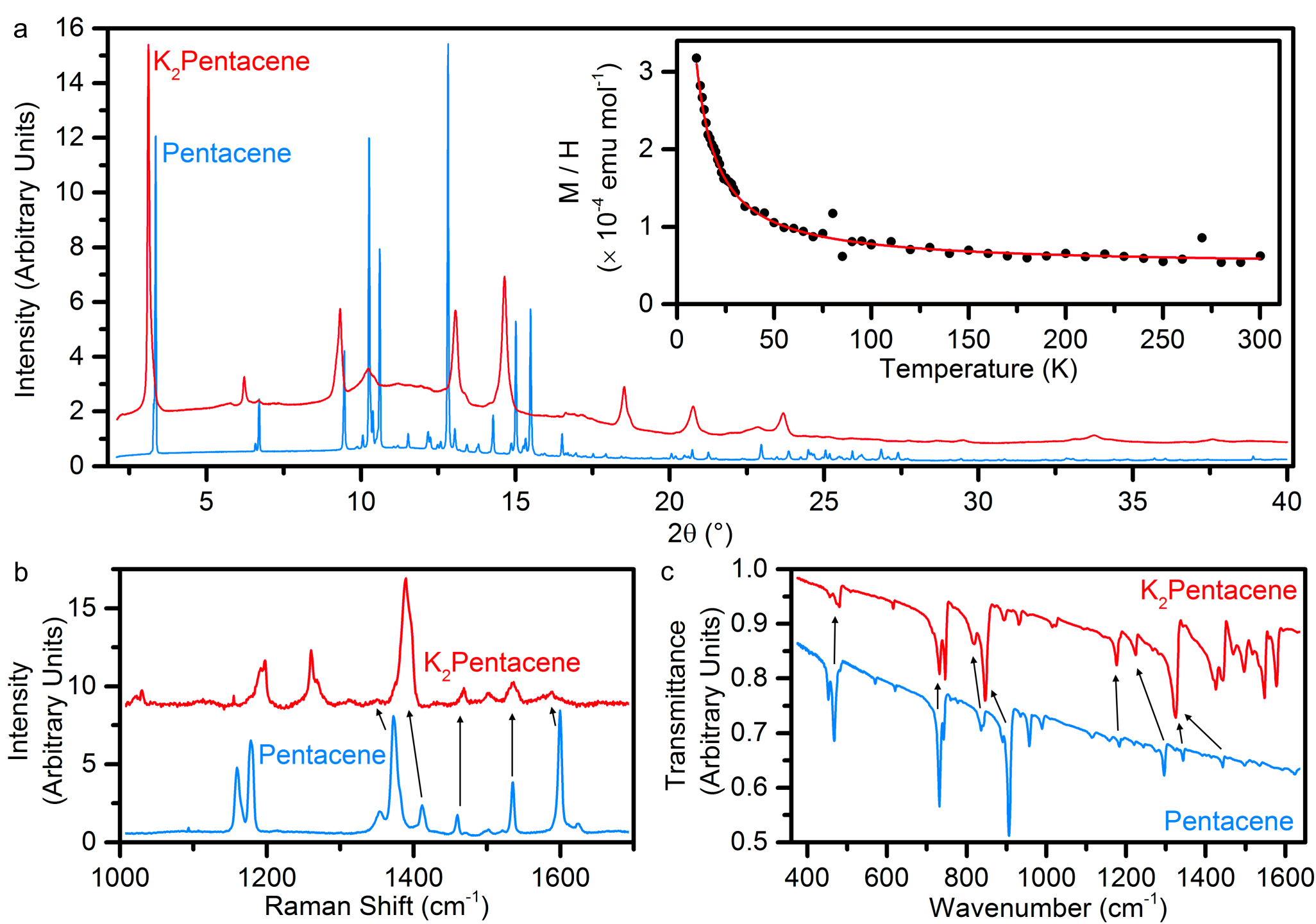
a

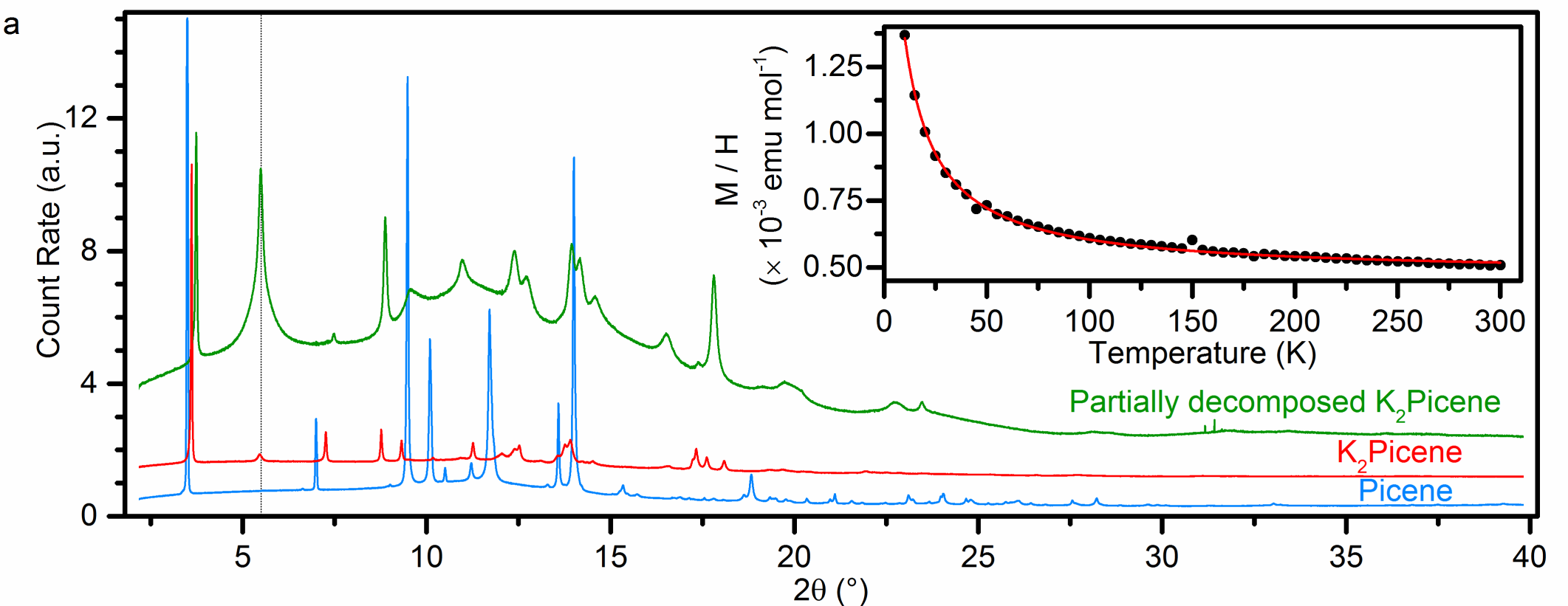

b

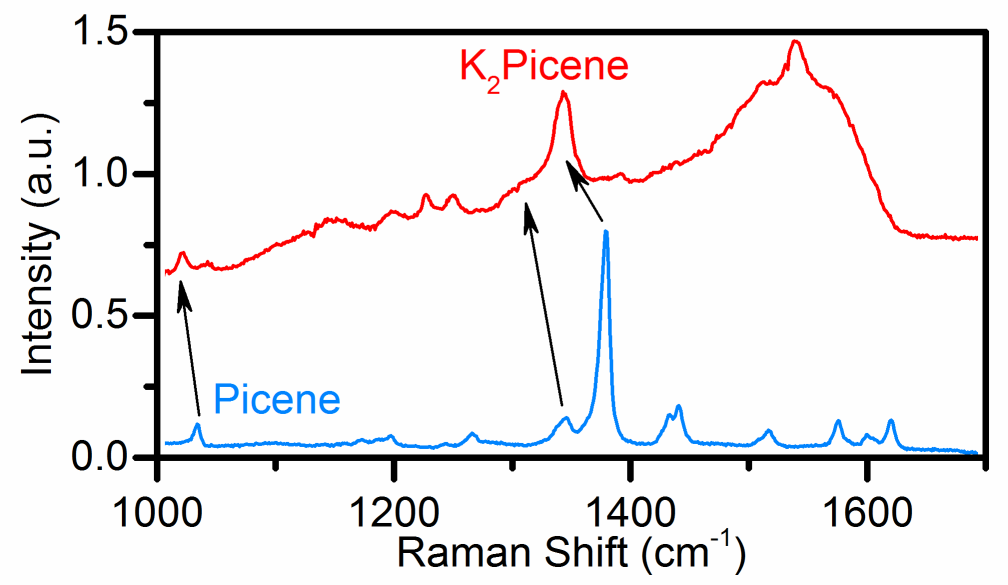

$c$

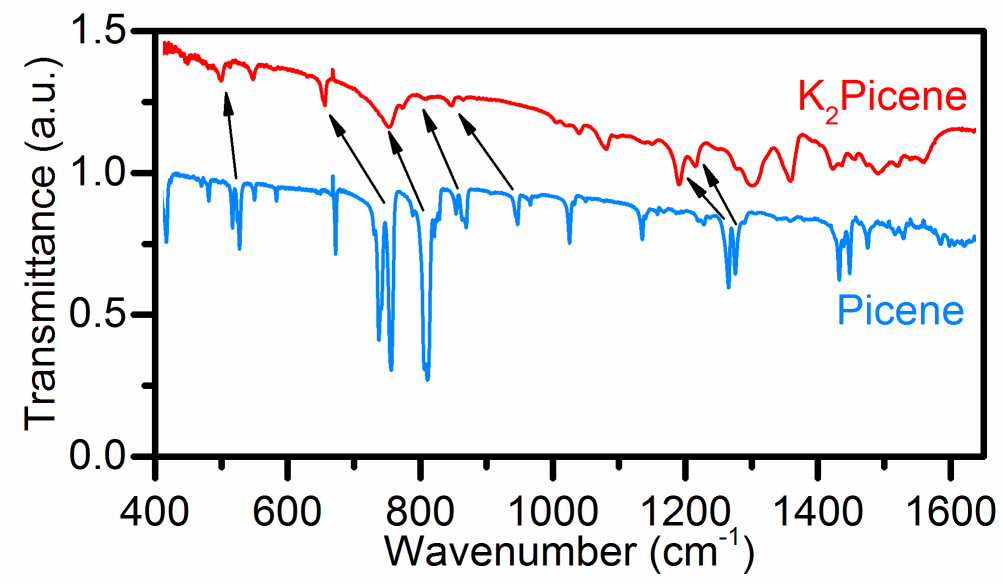



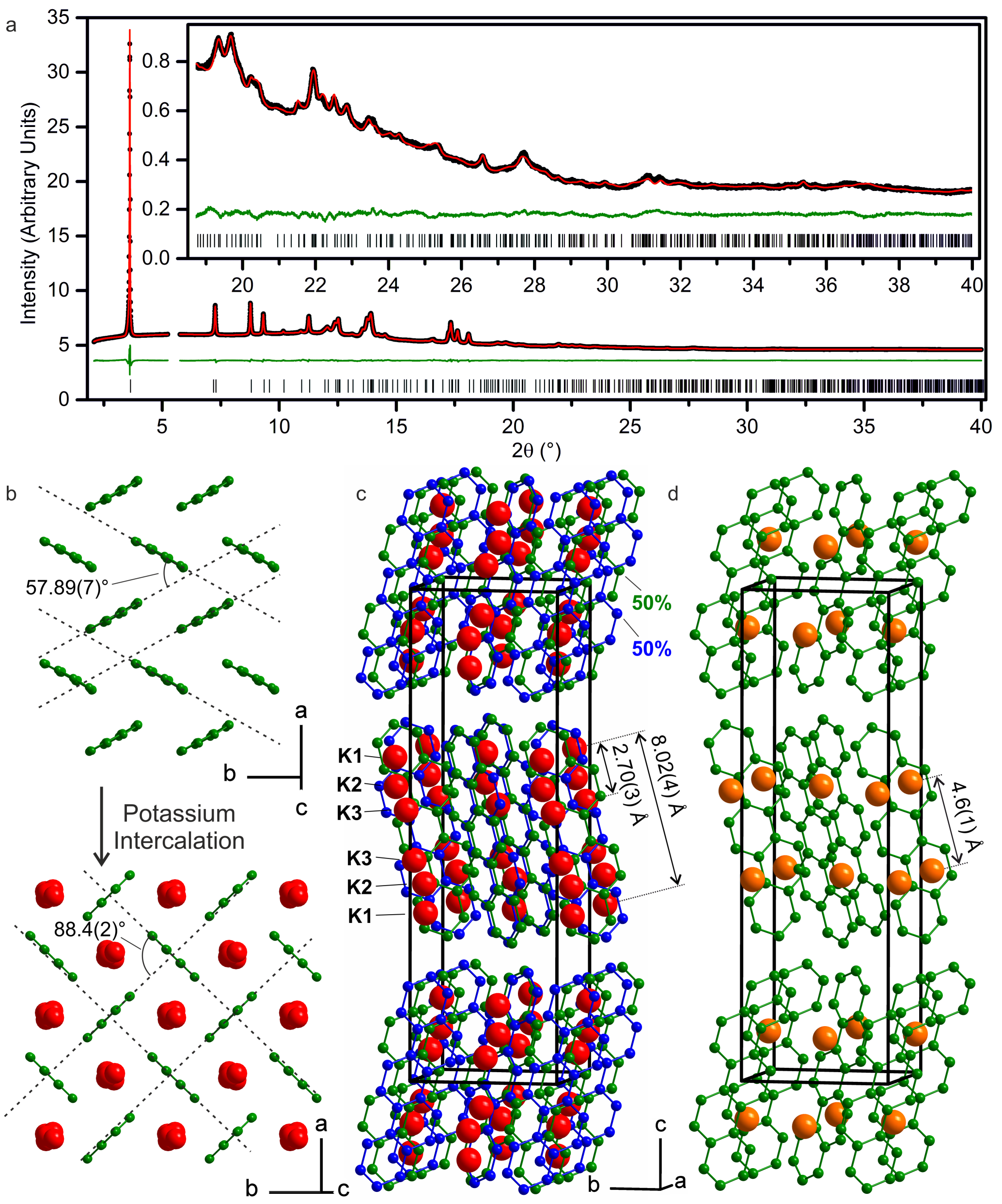
a

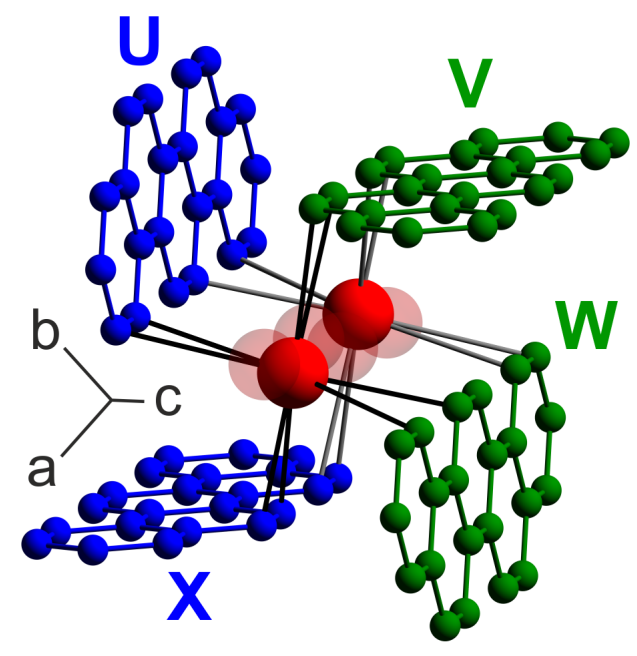

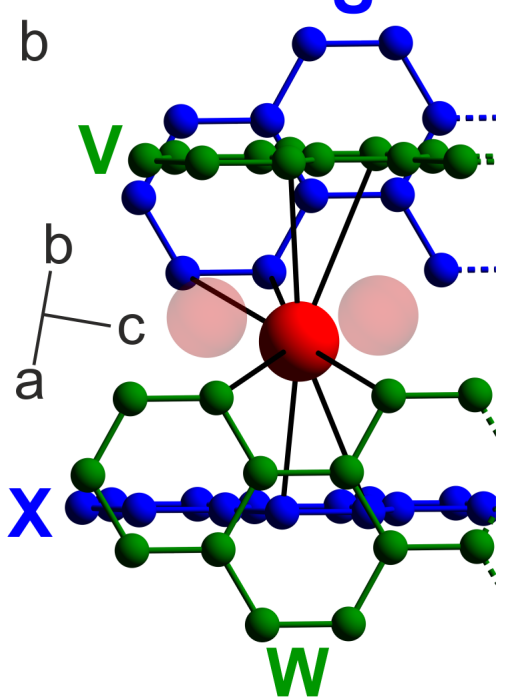

C
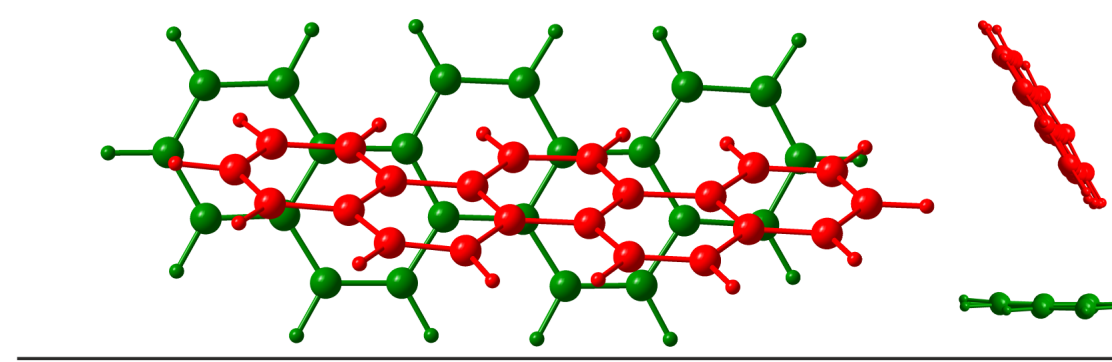

$=0-0$
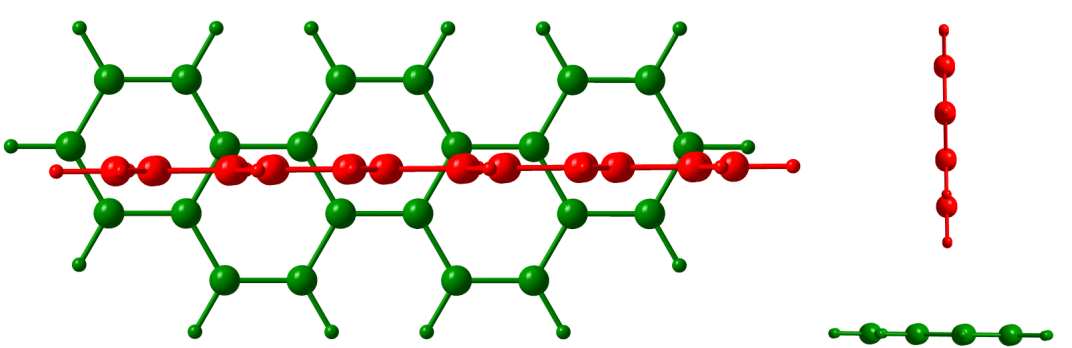

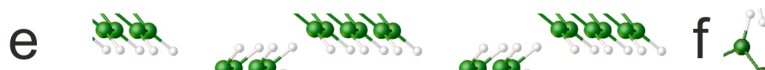

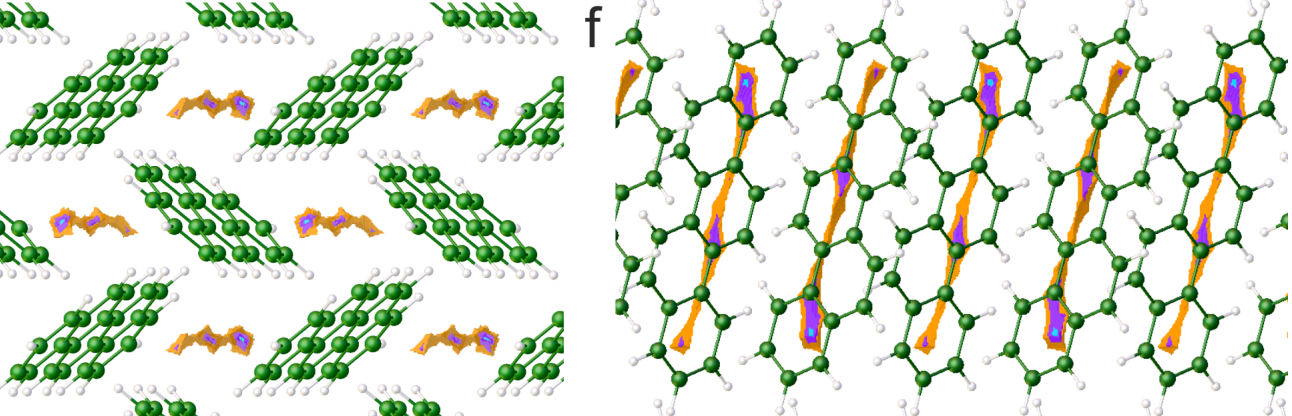

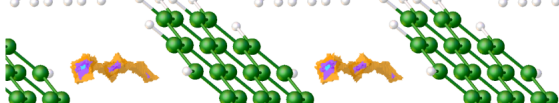
(a)

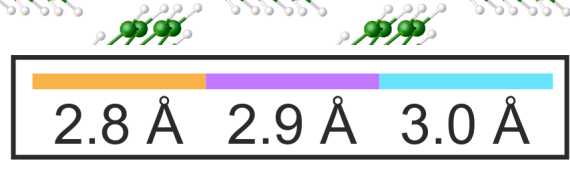

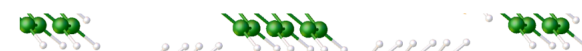

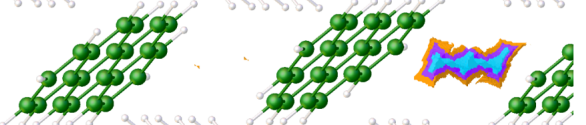

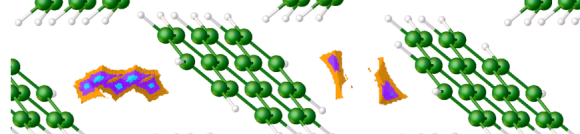

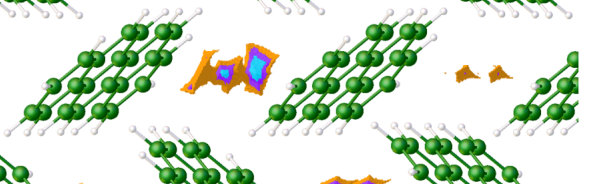
i) 200200

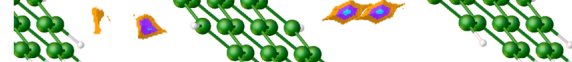
- vos gros gros of
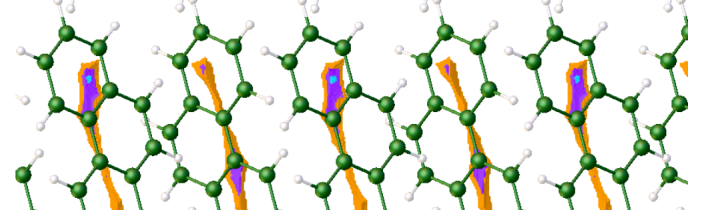

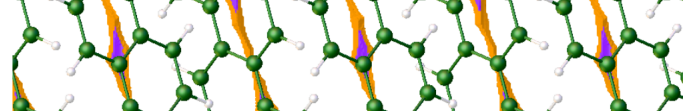

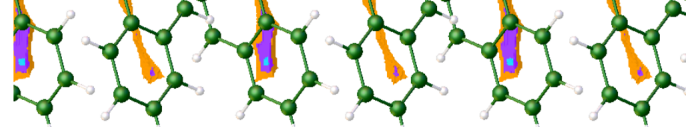
jo of of of of of

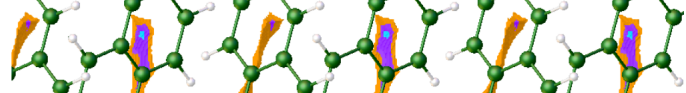
bo otoloto ofoloto.

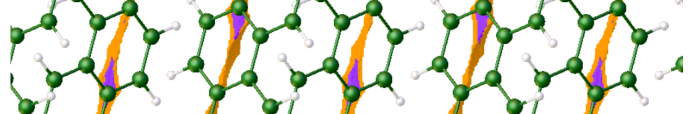

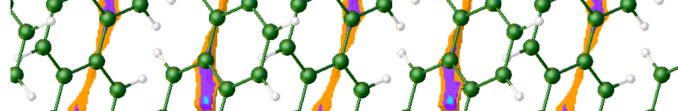

